\title{
Localisation des Espaces de Besov Homogènes
}

\section{ABDELLAH YOUSSFI}

Introduction. L'un des problèmes, qui remontent aux travaux de B. Muckenhoupt, R. L. Wheeden et W. S. Young [5], a été de caractériser (en dimension quelconque) les multiplicateurs de Fourier de $L^{2}\left(|x|^{2 s} d x\right)(s \in \mathbf{R})$. En travaillant dans cette direction, E. Sawyer [7] a remarqué que le problème revient à déterminer les multiplicateurs de $\dot{X}_{2}^{s, 2}$. L'espace $\dot{X}_{2}^{s, 2}$ contient un et un seul élément de chaque classe de l'espace $\dot{B}_{2}^{s, 2}$ (espace de Besov homogène); d'ailleurs G. Bourdaud [4] a récemment défini, de façon précise, les espaces $\dot{X}_{p}^{s, q}$ nommés "réalisation usuelle" de $\dot{B}_{p}^{s, q}$.

Dans son article, E. Sawyer a montré le théorème suivant :

Théorème 1. Soit $s>0$ vérifiant $s-\frac{n}{2} \in \mathbf{R}^{+} / \mathbf{N}$ et $\frac{s-n}{2} \in \mathbf{R}^{+} / \mathbf{N}$; alors les propriétés suivantes sont équivalentes :

(1) $m$ est un multiplicateur de $\dot{X}_{2}^{s, 2}$,

(2) $\sup _{t>0}\left\|\eta \cdot d_{t} m\right\|_{B_{2}^{s, 2}}<+\infty$; où $B_{p}^{s, q}$ désigne l'espace de Besov inhomogène, $\left(d_{t} m\right)(x)=m\left(\frac{x}{t}\right)^{2}$ et $\eta$ une fonction de classe $C^{\infty}$, portée par $\frac{1}{4} \leq|x| \leq 4$ et égale à 1 sur $\frac{1}{2} \leq|x| \leq 2$.

Comme il arrive en pareil cas, E. Sawyer n'a pas fourni une preuve simple à son théorème. Les techniques utilisées apparaissent compliquées pour pouvoir étendre le théorème aux espaces $\dot{X}_{p}^{s, p}$.

Un problème, adjacent au précédent, était de caractériser les multiplicateurs des espaces inhomogènes. Pour ce faire, le cas des espaces de Sobolev a conduit Stritchartz [9] à commencer par étudier la localisation de ces espaces. Peu après, J. Peetre [6] a amorcé l'étude des espaces de Besov.

A partir de là, G. Bourdaud [2] a donné une forme générale à la localisation, dans le cadre des espaces de Banach de distributions : on part d'une fonction $h \in D\left(\mathbf{R}^{n}\right)$ vérifiant : 


$$
f=\sum_{k \in Z^{n}}\left(\tau_{k} h \cdot f\right) \quad \text { pour toute distribution } f
$$

où $\tau_{k} h(x)=h(x-k)$.

Un espace de Banach de distributions est dit localisable en norme $\ell^{p}\left(\mathbf{Z}^{n}\right)$ si :

$$
\|f\| \approx\left[\sum_{k \in Z^{n}}\left\|\tau_{k} h \cdot f\right\|^{p}\right]^{1 / p}
$$

L'un des résultats substantiels est que la localisation en norme $\ell^{p}\left(\mathbf{Z}^{n}\right)$ de l'espace donne, automatiquement, la localisation en norme $\ell^{\infty}\left(\mathbf{Z}^{n}\right)$ de l'espace des multiplicateurs.

Par ailleurs, il est bien connu que l'étude des espaces fonctionnels repose sur la structure du groupe des translations et sur celle du groupe des dilatations. Notre object est ici, de remplacer dans le travail de G. Bourdaud, le groupe des translations par celui des dilatations. Ensuite on montrera que, dans ce cas, on aboutit aux mêmes résultats essentiels; c'est ce qui occupera la première partie.

La deuxième partie sera un rappel sur les espaces de Besov; dans les troisième et quatrième parties on s'intéressera à la localisation des espaces $\dot{X}_{p}^{s, p}$. C'est ainsi que dans la cinquiéme partie on obtiendra une généralisation du théorème de E. Sawyer avec une démonstration plus simple.

Finalement, dans le cadre des intégrales fractionnelles, on montrera une inégalité du type de celle de Hardy-Littlewood [8] pour les espaces $\dot{X}_{p}^{s, q}$ dans le cas $s-\frac{n}{p} \notin N, s>0$ et $p \geq q$.

En fin, je tiens à exprimer mes vifs remerciements à Mr. Gèrard Bourdaud pour l'intérêt qu'il a porté à ce travail; ses conseils et ses remarques m'ont été très précieux.

\section{Notation.}

$\mathbf{R}_{0}^{n}=$ l'ouvert $\mathbf{R}^{n} /\{0\}$.

$D\left(\mathbf{R}^{n}\right)=D=$ espace des fonctions de classe $C^{\infty}$ à support compact.

$D\left(\mathbf{R}_{0}^{n}\right)=$ le sous-espace de $D\left(\mathbf{R}^{n}\right)$ formé des fonctions à support contenu dans $\mathbf{R}_{0}^{n}$.

$S\left(\mathbf{R}^{n}\right)=$ espace de $S$ chwartz des fonctions $C^{\infty}$ à décroissance rapide.

$D^{\prime}\left(\mathbf{R}^{n}\right)=$ espace des distributions sur $\mathbf{R}^{n}$.

$D^{\prime}\left(\mathbf{R}_{0}^{n}\right)=$ le dual de $D\left(\mathbf{R}_{0}^{n}\right)$.

$S^{\prime}\left(\mathbf{R}^{n}\right)=$ espace des distributions tempérées.

$[f]=$ classe de $f$ modulo les polynômes.

$\hat{f}=$ transformée de Fourier de $f$.

$\|f\|_{p}$ désigne la norme de $f$ dans $L^{p}=L^{p}\left(\mathbf{R}^{n}\right)(1 \leq p \leq+\infty)$;

$p^{\prime}$ désigne l'exposant conjugué de $p, p^{\prime}=\frac{p}{p-1}$.

Si $\alpha=\left(\alpha_{1}, \ldots, \alpha_{n}\right) \in \mathbf{N}^{n},|\alpha|=\alpha_{1}+\cdots+\alpha_{n}$ et $f^{(\alpha)}=\partial_{x_{1}}^{\alpha_{1}} \ldots \partial_{x_{n}}^{\alpha_{n}} f$. 
La lettre $C$ désignera une constante positive dépendant de l'espace fonctionnel considéré, de certaines fonctions auxiliaires, etc...; sa valeur peut changer d'une occurrence à l'autre.

I. Généralités sur la localisation multiplicative. Rappelons qu'un espace de Banach de distributions $E$ (en abrégé E.B.D. ) est un sous-espace de $D^{\prime}\left(\mathbf{R}^{n}\right)$ muni d'une norme complète $\|\cdot\|_{E}$ qui rend continue l'injection canonique $E \rightarrow$ $D^{\prime}\left(\mathbf{R}^{n}\right)$.

Nous dirons que $E$ est un $D$-module (resp. $D\left(\mathbf{R}_{0}^{n}\right)$-module) si les éléments de $D\left(\mathbf{R}^{n}\right)$ (resp. de $D\left(\mathbf{R}_{0}^{n}\right)$ ) opérent sur $E$ par multiplication.

Choisissons une fonction auxiliaire $\eta \in D\left(\mathbf{R}_{0}^{n}\right)$ (fixée une fois pour toutes), portée par $\frac{1}{2} \leq|x| \leq 2$ telle que : $\sum_{j \in Z} \eta\left(2^{j} x\right)=1$ pour $x \neq 0$. Ainsi, pour toute distribution $f$ on a :

$$
f=\sum_{j \in \mathbf{Z}}\left(\eta\left(2^{j}(\cdot)\right) f\right) \quad \text { dans } D^{\prime}\left(\mathbf{R}_{0}^{n}\right) .
$$

Dès lors, nous nous intéressons aux E.B.D.. $E$ tels que $E$ soit un $D\left(\mathbf{R}_{0}^{n}\right)-$ module et pour toute $f \in E$, on puisse identifier $f$ et $\sum_{j}\left(n\left(2^{j}(\cdot) f\right)\right.$ dans $D^{\prime}\left(\mathbf{R}^{n}\right)$. Ce qui nous conduit,aussitôt, à travailler avec des sous-espaces de $D^{\prime}\left(\mathbf{R}_{0}^{n}\right)$.

Définition 1. On appelle espace de Banach de distributions de $\mathbf{R}_{0}^{n}$ (en abrégé E.B.D. ( $\left.\left.\mathbf{R}_{0}^{n}\right)\right)$ tout sous-espace $E$ de $D^{\prime}\left(\mathbf{R}_{0}^{n}\right)$ muni d'une norme complète $\|\cdot\|_{E}$ qui rend continue l'injection canonique $E \rightarrow D^{\prime}\left(\mathbf{R}_{0}^{n}\right)$.

Consideróns le groupe des homothéties $d_{\lambda}: x \rightarrow \lambda x(\lambda>0)$; l'action de $d_{\lambda}$ sur $D^{\prime}\left(\mathbf{R}_{0}^{n}\right)$ est définie par :

$$
d_{\lambda} \varphi(x)=\varphi\left(\frac{x}{\lambda}\right)
$$

si $\varphi \in D\left(\mathbf{R}_{0}^{n}\right)$ et $\left\langle d_{\lambda} u, \varphi\right\rangle=\lambda^{n}\left\langle u, d_{\lambda-1} \varphi\right\rangle$ pour $u \in D^{\prime}\left(\mathbf{R}_{0}^{n}\right)$.

Définition 2. Soit $E$ un E.B.D. $\left(\mathbf{R}_{0}^{n}\right), E$ est homogène de degrè $\alpha \in \mathbf{R}$ si pour tout $f \in E$ et tout $\lambda>0, d_{\lambda} f \in E$ et $\left\|d_{\lambda} f\right\|_{E}=\lambda^{\alpha}\|f\|_{E}$.

Proposition 1. Soit $E$ un E.B.D. ( $\left.\mathbf{R}_{0}^{n}\right)$, homogène de degré $\alpha$, et qui soit un $D\left(\mathbf{R}_{0}^{n}\right)$-module, alors on peut trouver $C>0$ et $N \in \mathbf{N}$ tels que, pour toute fonction $\varphi \in D\left(\mathbf{R}^{n}\right)$ avec $\varphi(0)=0$ et tout $f \in E$ on ait $: \varphi \cdot f \in E$ et

$$
\|\varphi f\|_{E} \leq C\|f\|_{E}\left[\sup _{\substack{|\gamma| \leq N \\ x \in \mathbf{R}^{n}}}(1+|x|)^{N}\left|\varphi^{(\gamma)}(x)\right|\right] .
$$


Preuve : Soit $K=\left\{\frac{1}{2} \leq|x| \leq 2\right\}$; il existe (voir [2], Proposition 1) alors $C>0$ et $N \in N$ tels que :

$$
\|\psi \cdot f\|_{E} \leq C\|f\|_{E} \sup _{|\gamma| \leq N}\left\|\psi^{(\gamma)}\right\|_{\infty}
$$

pour $\psi \in D\left(\mathbf{R}_{0}^{n}\right)$ portée par $K$ et $f \in E$.

Supposons $\psi \in D\left(\mathbf{R}_{0}^{n}\right)$, portée par $\frac{r}{2} \leq|x| \leq 2 r(r>0)$; on applique, successivement, l'homogénéité de $E$ et (2) à $d_{r}-1 \psi$ on obtient :

$$
\|\psi \cdot f\|_{E} \leq C\|f\|_{E} \sup _{|\gamma| \leq N} r^{|\gamma|}\left\|\psi^{(\gamma)}\right\|_{\infty} \quad \text { pour tout } f \in E .
$$

Soient, maintenant, $\varphi \in D\left(\mathbf{R}^{n}\right)$ avec $\varphi(0)=0$ et $f \in E$, alors $\varphi f \in D^{\prime}\left(\mathbf{R}_{0}^{n}\right)$ et $\varphi f=\sum_{j \in \mathbf{Z}}\left(\eta\left(2^{j}(\cdot)\right) \varphi f\right)$ dans $D^{\prime}\left(\mathbf{R}_{0}^{n}\right)$; de sorte que la convergence normale dans $E$ de la série $\sum_{j \in \mathbf{Z}}\left(\eta\left(2^{j}(\cdot)\right) \varphi f\right)$, nous donnera : $\varphi f \in E$ et $\|\varphi f\|_{E} \leq A+B$ où

$$
A=\sum_{j \geq 1}\left\|\eta\left(2^{-j}(\cdot)\right) \varphi f\right\|_{E}
$$

et

$$
B=\sum_{j \leq 0}\left\|\eta\left(2^{-j}(\cdot)\right) \varphi f\right\|_{E}
$$

Majoration de $A$. En appliquant (3) à chacun des termes de la série on obtient :

$$
A \leq C\|f\|_{E}\left\{\sum_{j \geq 1}\left(\sup _{|\gamma| \leq N}\left[2^{j|\gamma|}\left\|\left(\eta\left(2^{-j}(\cdot)\right) \varphi\right)^{(\gamma)}\right\|_{\infty}\right]\right)\right\} .
$$

Alors, le fait que $|x| \approx 2^{j}$ sur le support de $\eta\left(2^{-j}(\cdot)\right) \varphi$ et la formule de Leibnitz nous prouvent :

$$
\sup _{|\gamma| \leq N}\left[2^{j|\gamma|}\left|\left(\eta\left(2^{-j}(\cdot)\right) \varphi\right)^{(\gamma)}(x)\right|\right] \leq C \cdot 2^{-j} \sup _{\substack{|\gamma| \leq N \\|x| \geq 1}}\left[|x|^{N+1}\left|\varphi^{(\gamma)}(x)\right|\right]
$$

pour $j \geq 1$.

Finalement, $A$ est majorée par $C\|f\|_{E} \sup _{\substack{|\gamma| \leq N \\ x \in \mathbf{R}^{n}}}\left[(1+|x|)^{N+1} \mid \varphi^{(\gamma)}(x) \|\right]$.

Majoration de $B$. De (3) on tire :

$$
B \leq C\|f\|_{E} \sum_{j \leq 0} \sup _{|\gamma| \leq N}\left(2^{j|\gamma|}\left\|\left(\eta\left(2^{-j}(\cdot)\right) \varphi\right)^{(\gamma)}\right\|_{\infty}\right) .
$$

Pour $1 \leq|\gamma| \leq N$, la formule de Leibnitz entraine :

$$
2^{j|\gamma|}\left|\left(\eta\left(2^{-j}(\cdot)\right) \varphi\right)^{(\gamma)}(x)\right| \leq C\left[2^{j} \sup _{|\beta| \leq N}\left\|\varphi^{(\beta)}\right\|_{\infty}+\left|\eta^{(\gamma)}\left(2^{-j} x\right) \varphi(x)\right|\right] .
$$


De plus, l'hypothèse $\varphi(0)=0$, nous donne :

$$
\left|\eta^{(\gamma)}\left(2^{-j} x\right) \varphi(x)\right| \leq C 2^{j}\|\nabla \varphi\|_{\infty}
$$

pour $|\gamma| \leq N$; ce qui montre que $B$ est majorée par

$$
C\|f\|_{E} \sup _{\substack{|\gamma| \leq N+1 \\ x \in \mathbf{R}^{n}}}\left[(1+|x|)^{N+1}\left|\varphi^{(\gamma)}(x)\right|\right]
$$

Remarque. Si $E$ satisfait les hypothèses de la Proposition 1, (1) entraîne que les fonctions de $S\left(\mathbf{R}^{n}\right)$ nulles à l'origine, opèrent par multiplication sur $E$. En posant $\|\varphi\|_{M(E)}=\sup \left\{\|\varphi f\|_{E},\|f\|_{E} \leq 1\right\}$, on obtient de plus : $\|\varphi\|_{M(E)} \leq$ $C \sup _{|\gamma| \leq N+1}\left[(1+|x|)^{N}\left|\varphi^{(\gamma)}(x)\right|\right]$ pour $\varphi \in S\left(\mathbf{R}^{n}\right)$ et $\varphi(0)=0$.

Corollaire 1. Sous les hypothèses de la proposition 1, pour toutes fonctions $\varphi \in D\left(\mathbf{R}_{0}^{n}\right)$ et $g \in S\left(\mathbf{R}^{n}\right)$ avec $g^{(\gamma)}(0)=0$ pour $|\gamma| \leq N$, on ait :

$$
\left\|g d_{\lambda} \varphi\right\|_{M(E)} \leq C \inf \left(\lambda, \lambda^{-1}\right) \quad \text { pour } \lambda>0
$$

En particulier, la suite $\left(\left\|g d_{2} j \varphi\right\|_{M(E)}\right)_{j \in Z}$ appartient à $\ell^{1}(\mathbf{Z})$.

Dans la suite l'E.B.D. $\left(\mathbf{R}_{0}^{n}\right), E$ sera un $D\left(\mathbf{R}_{0}^{n}\right)$-module, homogène.

Définition 3. Pour $p \in[1,+\infty]$, on désigne par $(E)_{\ell^{p}(\mathbf{z})}$ l'espace des distributions $f \in D^{\prime}\left(\mathbf{R}_{0}^{n}\right)$ telles que : $\left(\eta\left(2^{j}(\cdot)\right) f\right) \in E$ pour tout $j \in \mathbf{Z}$ et

$$
\|f\|_{(E)_{\ell P(\mathbf{Z})}}=\|\left(\left\|\eta\left(2^{j}(\cdot)\right) f\right\|_{E)_{j \in \mathbf{Z}} \|_{\ell p(\mathbf{Z})}<+\infty}\right.
$$

On dit que $E$ est localisable en norme $\ell^{p}(\mathbf{Z})$ si $E=(E)_{\ell^{p}}(\mathbf{Z}) \cdot(E)_{\ell^{p}}(\mathbf{Z})$ est un E.B.D. ( $\left.\mathbf{R}_{0}^{n}\right)$ qui ne dépend pas du choix de la fonction $\eta$. Ceci est une conséquence du lemme suivant :

Lemme 1. Pour tout $\gamma>1$, il existe $C>0$ tel que, pour toute suite $\left(f_{j}\right)_{j \in \mathbf{Z}}$ d'éléments de $E$, portés respectivement par $\gamma^{-1} 2^{j} \leq|x| \leq \gamma 2^{j}$ on ait :

$$
\left\|\sum_{j \in \mathbf{Z}} f_{j}\right\|_{(E)_{\ell P(\mathbf{Z})}} \leq C\left\|\left(\left\|f_{j}\right\|_{E}\right)_{j}\right\|_{\ell P(\mathbf{Z})} .
$$


Preuve: On pose $f=\sum_{j \in \mathbf{Z}} f_{j}$ alors $\eta\left(2^{-k}(\cdot)\right) f=\sum_{|k-j| \leq M} \eta\left(2^{-k}(\cdot)\right) f_{j}$ où $M=\left[\log _{2}(\gamma)\right]+1$. Donc

$$
\left\|\eta\left(2^{-k}(\cdot)\right) f\right\|_{E} \leq \sum_{\nu=-M}^{M}\left\|\eta\left(2^{-k}(\cdot)\right)\right\|_{M(E)}\left\|f_{k+\nu}\right\|_{E} .
$$

La preuve se termine en remarquant que :

$$
\left\|d_{\lambda} \eta\right\|_{M(E)}=\|\eta\|_{M(E)} \quad \text { pour tout } \lambda>0 .
$$

Comme dans [2], pour la localisation additive, on peut définir $(E)_{\ell p(\mathbf{Z})}$ en remplaçant la fonction $\eta$ par certaines fonctions de $S\left(\mathbf{R}^{n}\right)$ :

Proposition 2. Soit $\vartheta \in S\left(\mathbf{R}^{n}\right)$ telle que $\vartheta^{(\gamma)}(0)=0$ pour $|\gamma| \leq N$ et $\vartheta \neq 0$ sur le support de $\eta$, alors :

$$
\|f\|_{(E)_{\ell P(\mathbf{Z})}} \approx\left[\sum_{j \in \mathbf{Z}}\left\|\vartheta\left(2^{j}(\cdot)\right) f\right\|_{E}^{p}\right]^{1 / p} .
$$

Preuve : D'une part, on peut écrire $\eta=\vartheta \cdot g$ où $g \in D\left(\mathbf{R}_{0}^{n}\right)$, donc

$$
\begin{aligned}
\left\|\eta\left(2^{j}(\cdot)\right) f\right\|_{E} & \leq\left\|g\left(2^{j}(\cdot)\right) \vartheta\left(2^{j}(\cdot)\right) f\right\|_{E} \\
& \leq\|g\|_{M(E)}\left\|\vartheta\left(2^{j}(\cdot)\right) f\right\|_{E}
\end{aligned}
$$

d'où :

$$
\|f\|_{(E)_{\ell p(\mathbf{z})}} \leq\|g\|_{M(E)} \|\left(\left\|\vartheta\left(2^{j}(\cdot) f \|_{E}\right)_{j}\right\|_{1^{p}(\mathbf{Z})} .\right.
$$

Inversement, soit $\chi \in D\left(\mathbf{R}^{n}\right)$, portée par $\frac{1}{4} \leq|x| \leq 4$ telle que $\chi=1$ sur $\frac{1}{2} \leq|x| \leq 2$

$$
\begin{aligned}
\left\|\vartheta\left(2^{k}(\cdot)\right) f\right\|_{E} & \leq \sum_{j \in \mathbf{Z}}\left\|\vartheta\left(2^{k}(\cdot)\right) \eta\left(2^{j}(\cdot)\right) f\right\|_{E} \\
& \leq \sum_{j \in \mathbf{Z}}\left\|\vartheta\left(2^{k}(\cdot)\right) \chi\left(2^{j}(\cdot)\right)\right\|_{M(E)} \| \eta\left(2^{j}(\cdot) f \|_{E} .\right.
\end{aligned}
$$

L'inégalité s'obtient en conbinant l'inégalité de Young et le corollaire 1.

De la proposition suivante, il résultera, entre autres, une condition suffisante pour que l'espace ne soit localisable en aucune norme $\ell^{p}(\mathbf{Z})$ :

Proposition 3. Il existe $p_{1}$ et $p_{2}, 1 \leq p_{1} \leq p_{2} \leq \infty$ vérifiant :

(1) $\operatorname{Si}(E)_{\ell^{P}(\mathbf{Z})} \subseteq E$ alors $p \leq p_{1}$;

(2) Si $E \subseteq(E)_{\ell p(z)}$ alors $p \geq p_{2}$;

(3) Si $E$ est localisable en norme $\ell^{p}(Z)$ alors $p=p_{1}=p_{2}$. 
Preuve : Par hypothèse sur $E$ on a $E \subseteq(E)_{\ell^{\infty}(\mathbf{z})}$ et $(E)_{\ell^{1}(\mathbf{Z})} \subseteq E$; donc les ensembles $\left\{p \geq 1, E \subseteq(E)_{\ell^{p}(\mathbf{z})}\right\}$ et $\left\{p \geq 1,(E)_{\ell^{p}(\mathbf{Z})} \subseteq E\right\}$ sont non vides et sont des intervalles d'après la croissance de $\ell^{p}(\mathbf{Z})$ en $p$.

On pose :

$$
p_{1}=\sup \left\{p \geq 1,(E)_{\ell p(\mathbf{Z})} \subseteq E\right\}
$$

et

$$
p_{2}=\inf \left\{p \geq 1, E \subseteq(E)_{\ell^{p}(\mathbf{z})}\right\}
$$

Alors, modulo la croissance stricte de $(E)_{\ell p(z)}$ en $p$, il est facile de voir que $p_{1}$ et $p_{2}$ vérifient les propriétés souhaitées.

Pour montrer que $(E)_{\ell p(\mathbf{z})}$ croît strictement en $p$, on utilise la croissance stricte de $\ell^{p}(\mathbf{Z})$ en $p$, le lemme 1 et le résultat suivant :

Lemme 2. Pour tous a et $b$ avec $0<a<b<2 a$, on peut trouver $C>0$ tel que, pour toute suite $\left(f_{j}\right)_{j \in \mathbf{Z}}$ d'éléments de $E$ portés respectivement par $a 2^{j} \leq|x| \leq b 2^{j}$ on ait :

$$
\left\|\left(\left\|f_{j}\right\|_{E}\right)\right\|_{\ell^{p}(\mathbf{Z})} \leq C\left\|\sum_{j \in \mathbf{Z}} f_{j}\right\|_{(E)_{\ell^{p}(\mathbf{Z})}} .
$$

Preuve : On considère $\chi \in D\left(\mathbf{R}^{n}\right)$ portée par $\alpha \leq|x| \leq \beta$ telle que $\chi=1$ sur $a \leq|x| \leq b$ où $\beta>2 \alpha$ et $\frac{b}{2}<\alpha<a<b<\beta<2 a$. Alors

$$
\chi\left(2^{-j}(\cdot)\right) f=f_{j}, \quad f=\sum_{j \in Z} f_{j} .
$$

La dernière propriété, de la proposition 3, s'exprime aussi, de la façon suivante :

Corollaire 2. S'il existe $p \in] 1,+\infty\left[\right.$ tel qu'on n'ait ni $E \subseteq(E)_{\ell^{p}(\mathbf{Z})}$, ni $(E)_{\ell^{p}(\mathbf{Z})} \subseteq E$, alors $E n^{\prime}$ 'st localisable en aucune norme $\ell^{r}(\mathbf{Z}), r \in[1,+\infty]$.

II. Rappels sur les espaces de Besov homogènes. On se donne une fonction $\varphi \in D\left(\mathbf{R}^{n}\right)$, portée par la couronne $\frac{1}{2} \leq|x| \leq 2$ telle que $\sum_{j \in Z} \varphi\left(2^{j} \xi\right)=1$ pour $\xi \neq 0$ (on peut supposer, au besoin, que $\varphi=1$ sur $\frac{3}{4} \leq|\xi| \leq \frac{5}{4}$ ).

La fonction $\psi(\xi)=1-\sum_{j \geq 1} \varphi\left(2^{-j} \xi\right)$ est de classe $C^{\infty}$, portée par la boule $|\xi| \leq 2$ et on a :

$$
\psi(\xi)+\sum_{j \geq 1} \varphi\left(2^{-j} \xi\right)=1 \quad \text { pour } \xi \in \mathbf{R}^{n}
$$

A cette partition de l'unité sont associés les opérateurs de convolution $\Delta_{j}$ et $S_{j}(j \in \mathbf{Z})$ de symbôles respectifs $\varphi\left(2^{-j} \xi\right)$ et $\psi\left(2^{-j} \xi\right)$. 
Pour toute distribution tempérée $f$ on $a$ :

$$
f=S_{k} f+\sum_{j \geq k+1} \Delta_{j} f \quad \text { dans } S^{\prime}\left(\mathbf{R}^{n}\right),(\forall k \in \mathbf{Z}) .
$$

Pour $s \in \mathbf{R}, p, q \in[1,+\infty]$, l'espace de Besov inhomogène $B_{p}^{s, q}$ est l'ensemble des distribution $f \in S^{\prime}\left(\mathbf{R}^{n}\right)$ telles que :

$$
\|f\|_{B_{p}^{q, q}}=\left\|S_{0} f\right\|_{p}+\left\|\left(2^{s j}\left\|\Delta_{j} f\right\|_{p}\right)_{j \geq 1}\right\|_{\ell^{q}(\mathrm{~N})}<+\infty
$$

Sa version homogène est l'espace $\dot{B}_{p}^{s, q}$ formé des distributions $f \in S^{\prime}\left(\mathbf{R}^{n}\right)$ telles que :

$$
\|f\|_{B_{p}^{,, q}}=\left\|\left(2^{s j}\left\|\Delta_{j} f\right\|_{p}\right)_{j \in Z}\right\|_{\ell q(Z)}<+\infty .
$$

$\dot{B}_{p}^{s, q}$ est un espace de distributions modulo les polynômes. De plus ni $\dot{B}_{p}^{s, q}$, ni $B_{p}^{s, q}$ ne dépendent du choix de la fonction $\varphi$. On remarque que dans le cas $s>0, B_{p}^{s, q}$ s'injecte continûment dans $\dot{B}_{p}^{s, q}$ par : $f \rightarrow[f],[f]=$ classe de $f$ modulo les polynômes (pour plus de détails, consulter par exemple J. Peetre [6] où H. Triebel [10]).

Suivant [4] on associe à chacun des espaces $\dot{B}_{p}^{s, q}$ un sous-espace $\dot{X}_{p}^{s, q}$ de $S^{\prime}\left(\mathbf{R}^{n}\right)$ nommè "rèalisation usuelle" de $\dot{B}_{p}^{s, q}$, qui lui est isomorphe. De plus, si $s-\frac{n}{p} \notin \mathrm{N}, \dot{X}_{p}^{s, q}$ est invariant par dilatations avec le même degré d'homogénéité; c'est d'ailleurs l'unique réalisation invariante par dilatations de $\dot{B}_{p}^{s, q}$.

\section{Le cas $s<\frac{n}{p}$}

On note par $\widetilde{C}_{0}$ l'ensemble des distributions $f$ tendant faiblement vers 0 à l'infini, c'est à dire $d_{\lambda} f$ tend vers 0 , dans $D^{\prime}\left(\mathbf{R}^{n}\right)$, quand $\lambda$ tend vers 0 :

$$
\dot{X}_{p}^{s, q}=\left\{f \in \tilde{C}_{0} \mid[f] \in \dot{B}_{p}^{s, q}\right\}
$$

$\dot{X}_{p}^{s, q}$ est aussie l'espace des distributions $f \in S^{\prime}\left(\mathbf{R}^{n}\right)$ telles que $f=\sum_{j \in \mathbf{Z}} \Delta_{j} f$ dans $S^{\prime}\left(\mathbf{R}^{n}\right)$ et $[f] \in \dot{B}_{p}^{s, q}$.

\section{Le $\operatorname{cas} s-\frac{n}{p} \in \mathbf{R}^{+} / \mathbf{N}$}

Soit $m=\left[s-\frac{n}{p}\right], \dot{X}_{p}^{s, q}$ est l'espace des fonctions $f$ qui vérifient les conditions suivantes :

- $f$ de classe $C^{m}$

- $f^{(\alpha)}(0)=0$ pour $|\alpha| \leq m$; 
- $\sup _{x \in \mathbf{R}^{n}}|x|^{(n / p+m-s)}\left|f^{(\alpha)}(x)\right|<+\infty$ pour $|\alpha|=m$;

- $[f] \in \dot{B}_{p}^{s, q}$.

Dans ce cas, $\dot{X}_{p}^{s, q}$ est l'espace formé des distributions $f \in S^{\prime}\left(\mathbf{R}^{n}\right)$ telles que, $[f] \in \dot{B}_{p}^{s, q}$ et $f=\sum_{j \in \mathbf{Z}}\left(\Delta_{j} f-\sum_{|\alpha| \leq m} \frac{1}{\alpha !}\left(\Delta_{j} f\right)^{(\alpha)}(0) \cdot x^{\alpha}\right)$ dans $S^{\prime}\left(\mathbf{R}^{n}\right)$.

Remarques. $p^{\prime}$ désigne l'exposant conjugué de $p$.

(i) Si $s<-\frac{n}{p}$, et $f \in \dot{X}_{p}^{s, q} \cap S\left(\mathbf{R}^{n}\right)$, alors $\int f(x) d x=0$; en particulier $\dot{X}_{p}^{s, q}$ n'est pas un $D\left(\mathbf{R}_{0}^{n}\right)$-module.

(ii) $\mathrm{Si}-\frac{n}{p^{\prime}}<s<\frac{n}{p}$ et $1 \leq p, q<+\infty$, on peut vérifier, sans peine, que $S\left(\mathbf{R}^{n}\right)$ est un sous-espace dense de $\dot{X}_{p}^{s, q}$; et le dual de $\dot{X}_{p}^{s, q}$ est $\dot{X}_{p^{\prime}}^{-s, q^{\prime}}$.

(iii) en utilisant l'interpolation des espaces de Besov (voir [6] ou [10]), $\dot{X}_{p}^{0, q}$ $(1<p, q<+\infty)$ s'obtient par interpolation complexe :

$$
\dot{X}_{p}^{0, q}=\left(X_{p_{1}}^{s, q}, X_{p_{2}}^{-s, q}\right)_{[\vartheta]}
$$

où

$$
\begin{aligned}
& 0<\vartheta<1, \\
& -\frac{n}{p_{1}^{\prime}}<s<\frac{n}{p_{1}}, \\
& -\frac{n}{p_{2}}<s<\frac{n}{p_{2}^{\prime}}, \quad \text { et } \\
& \frac{1}{p}=\frac{\vartheta}{p_{2}}+\frac{1-\vartheta}{p_{1}} .
\end{aligned}
$$

Proposition 4. Pour $-\frac{n}{p^{\prime}}<s<\frac{n}{p}$, où $s-\frac{n}{p} \in \mathbf{R}^{+} / \mathbf{N}, \dot{X}_{p}^{s, q}$ est un $D$-module, homogène de degré $\frac{n}{p}-s$ et l'application $J(u)=u / \mathbf{R}_{0}^{n}$ est injective de $\dot{X}_{p}^{s, q}$ dans $D^{\prime}\left(\mathbf{R}_{0}^{n}\right)$.

Preuve : Par dualité et interpolation, on se ramène à étudier le cas $0<s<$ $\frac{n}{p}$ et le cas $s-\frac{n}{p} \in \mathbf{R}^{+} / \mathbf{N}$.

Commençons par vérifier les propriétés de la réalisation :

1. Le $\operatorname{cas} 0<s<\frac{n}{p}$

Si $f \in \dot{X}_{p}^{s, q}$ et $g \in D\left(\mathbf{R}^{n}\right)$ alors $g f$ est une distribution à support compact, donc $g f \in \widetilde{C}_{0}$. 


\section{Le cas $s-\frac{n}{p} \in \mathbf{R}^{+} / \mathbf{N}: m=\left[s-\frac{n}{p}\right]$.}

Pour $f \in \dot{X}_{p}^{s, q}$ et $g \in D\left(\mathbf{R}^{n}\right), g f \in C^{m}$ et $(g f)^{(\alpha)}(0)=0$ pour $|\alpha| \leq m$; de plus si $|x| \geq 1$ on $a$ :

$$
\left|(g f)^{(\alpha)}(x)\right| \leq C|x|^{(s-n / p-n)} \quad \text { pour }|\alpha|=m
$$

Si $|x| \leq 1$, la formule de Leibnitz nous donne :

$$
\left|(g f)^{(\alpha)}(x)\right| \leq C \sum_{\gamma \leq \alpha}\left|g^{(\gamma)}(x)\right|\left|f^{(\alpha-\gamma)}(x)\right|
$$

si $|\gamma|=0,\left|f^{(\alpha)}(x)\right| \leq C|x|^{(s-n / p-m)}$ pour $|\alpha|=m$; et si $|\gamma| \neq 0$ on a :

$$
\begin{aligned}
\left|f^{(\alpha-\gamma)}(x)\right| & \leq C|x| \sup _{\substack{|y| \leq 1 \\
\left|\gamma^{\prime}\right| \leq m}}\left|f^{\left(\gamma^{\prime}\right)}(y)\right| \\
& \leq C|x|^{(s-n / p-m)}
\end{aligned}
$$

donc $\left|(g f)^{(\alpha)}(x)\right| \leq C|x|^{(s-n / p-m)}$ pour $|\alpha|=m$. Pour estimer la norme, on remarque que dans les deux cas $f$ s'écrit $f=f_{1}+f_{2}$ où $f_{1} \in B_{p}^{s, q}$ et $f_{2} \in C^{\infty}$; donc $g f_{1} \in B_{p}^{s, q}$ et $g f_{2} \in D\left(\mathbf{R}^{n}\right)$, a fortiori $[g f] \in \dot{B}_{p}^{s, q}($ car $s>0)$.

Pour $-\frac{n}{p^{\prime}}<s<\frac{n}{p}$ ou $s-\frac{n}{p} \in \mathbf{R}^{+} \mathbf{N}, \dot{X}_{p}^{s, q}$ s'identifie à un sous-espace de $D^{\prime}\left(\mathbf{R}_{0}^{n}\right)$ qui est un $D$-module, homogène. Désormais on se limite à ces deux cas et on cherche à étudier la localisation de $\dot{X}_{p}^{s, q}$. Pour cela, il nous sera utile d'énoncer le lemma de presque-orthogonalité suivant :

\section{Lemme 3}

(1) Pour tout $\gamma>1$, il existe $C>0$ tel que, pour toute suite $\left(f_{j}\right)_{j \in \mathrm{Z}}$ de fonctions à spectres respectifs dans $\gamma^{-1} 2^{j} \leq|\xi| \leq \gamma 2^{j}$ on ait :

$$
\left\|\sum_{j \in \mathbf{Z}} f_{j}\right\|_{B_{p}^{s, q}} \leq C\left[\sum_{j \in \mathbf{Z}} 2^{s j q}\left\|f_{j}\right\|_{p}^{q}\right]^{1 / q} .
$$

(2) Pour tous a et $b, 0<a<b<2 a$, il existe $C>0$ tel que, pour toute suite de fonctions $\left(f_{j}\right)_{j \in \mathbf{Z}}$ à spectres respectifs dans $a 2^{j} \leq|\xi| \leq b 2^{j}$ on ait :

$$
\left[\sum_{j \in \mathbf{Z}}\left(2^{s j}\left\|f_{j}\right\|_{p}\right)^{q}\right]^{1 / q} \leq C\left\|\sum_{j \in \mathbf{Z}} f_{j}\right\|_{\dot{B}_{p}^{s, q}} .
$$

(3) Pour $s>0$, on peut remplacer dans (1), les couronnes par les boules $|\xi| \leq \gamma 2^{j}$. 
Preuve : Ce lemme est connu pour les espaces inhomogènes (voir par exemple [1]). De plus $\left(1^{\circ}\right)$ et $\left(2^{\circ}\right)$ se prouvent de la même façon que pour les espaces inhomogènes. La preuve du $\left(3^{\circ}\right)$ se déduit facilement d'une inégalité qu'on utilisera dans d'autres circonstances; elle-même conséquence de l'inegalité de Young dans $\ell^{p}(\mathbf{Z})$ :

Lemme 4. Pour tout $a>1$ et toute suite $\left(\varepsilon_{j}\right)_{j \in Z} \in \ell^{q}(\mathbf{Z})$, la suite $\eta_{k}=a^{k} \sum_{j \geq k} a^{-j} \varepsilon_{j}$ vérifie :

$$
\left\|\left(\eta_{k}\right)_{k \in \mathbf{Z}}\right\|_{\ell^{q}(\mathbf{Z})} \leq \frac{a}{a-1}\left\|\left(\varepsilon_{j}\right)_{j}\right\|_{\ell^{q}(\mathbf{Z})} .
$$

III. Théorème de localisation. Le cas le plus simple des E.B.D. ( $\left.\mathbf{R}_{0}^{n}\right)$ est celui des espaces $L^{p}$, qui sont localisables en norme $\ell^{p}(\mathbf{Z})$; pour $\dot{X}_{p}^{s, q}$ on a :

\section{THÉORÈME 2}

$$
S i-\frac{n}{p^{\dagger}}<s<\frac{n}{p} \text { ou } s-\frac{n}{p} \in \mathbf{R}^{+} / \mathbf{N} \text { alors : }
$$

$$
\begin{array}{ll}
\dot{X}_{p}^{s, q} \subseteq\left(\dot{X}_{p}^{s, q}\right)_{\ell p(Z)} & \text { pour } p \geq q, \\
\left(\dot{X}_{p}^{s, q}\right)_{\ell p(Z)} \subseteq \dot{X}_{p}^{s, q} & \text { pour } p \leq q .
\end{array}
$$

En particulier $\dot{X}_{p}^{s, p}$ est localisable en norme $\ell^{p}(\mathbf{Z})$.

La preuve du théorème repose, entre autres, sur les lemmes suivants :

Lemme 5. Soit $\lambda \in \dot{B}_{1}^{-8,1}, \hat{\lambda} \neq 0$ sur $\frac{1}{2} \leq|\xi| \leq 2$; on pose $\lambda_{j}(x)=$ $2^{n j} \lambda\left(2^{j} x\right)$ et $L_{j}(f)=\lambda_{j} * f$, alors :

$$
\|f\|_{\dot{B}_{p}^{s, q}} \approx\left[\sum_{j} 2^{s j q}\left\|L_{j}(f)\right\|_{p}^{q}\right]^{1 / q}
$$

Le lemme 5 est démontré par J. Peetre [6] (pp. 155-158).

Lemme 6. Si $0<s<\frac{n}{p}$ ou $s-\frac{n}{p} \in \mathbf{R}^{+} / \mathbf{N}$, alors il existe $C>0$ tel que, pour toute $h \in \dot{X}_{p}^{s, q}$ portée par $2^{k-1} \leq|x| \leq 2^{k+1}$ on $a: h \in L^{p}$ et

$$
\|h\|_{p} \leq C 2^{s k}\|h\|_{\dot{X}_{p}^{s, q}}
$$

Preuve : Par homogénéité on se ramène au cas $k=0$; ce dernier découle facilement des propriétés de la réalisation décrites dans [3]. 
Lemme 7. Pour tout $N \in \mathrm{N}$ et tous a et $b, 0 \leq a<b$ et $r>0$, il existe une fonction $\vartheta \in S\left(\mathbf{R}^{n}\right)$ vérifiant :

(i) $\hat{\vartheta}$ est portée par $a \leq|\xi| \leq b$;

(ii) $\vartheta^{(\alpha)}(0)=0$ pour $|\alpha| \leq N$;

(iii) $\vartheta \neq 0$ sur $0<|x| \leq r$.

Preuve : Soient $\varepsilon>0$ (assez petit) et $f$ une fonction radiale portée par $|x| \leq 1$ telle que $\int f(x) x^{\alpha} d x=0,|\alpha| \leq N ; \hat{f}$ étant une fonction analytique et radiale, il existe $\varepsilon^{\prime}>0$ tel que $\hat{f}(\xi) \neq 0$ pour $0<|\xi|<\varepsilon^{\prime}$.

Si $t=\inf \left(\varepsilon, \frac{\varepsilon^{\prime}}{r}\right)$, la fonction $g(x)=\hat{f}(t x)$ vérifie $: g \neq 0$ sur $0<|x| \leq r$ et $\hat{g}$ est portée par $|\xi| \leq \varepsilon$. On choisit $\varepsilon<\frac{b-a}{2}$ et $a+\varepsilon \leq b^{\prime} \leq b+\varepsilon$, alors la fonction

$$
\vartheta(x)=e^{i b^{\prime} x_{1}} g(x)
$$

fait l'affaire.

\section{PREUVE DU THÉORÈME 2}

\section{A. Le cas $p \geq q$ :}

Prenons dans le lemme $7 a=\frac{1}{2}, b=r=2$ et $N$ assez grand, de sorte que par la proposition 2 , on ait :

$$
\|f\|_{\left(\dot{X}_{p}^{s, q}\right)_{\ell p}(\mathbf{z})} \approx\left[\sum_{k}\left\|\vartheta\left(2^{k}(\cdot)\right) f\right\|_{\dot{X}_{p}^{s, q}}^{p}\right]^{1 / p}
$$

1. Le cas $-\frac{n}{p^{\prime}}<s<\frac{n}{p}$ :

Pour toute $f \in \dot{X}_{p}^{s, q}$, on a $f=\sum_{j} \Delta_{j} f$ dans $S^{\prime}\left(\mathbf{R}^{n}\right)$, on peut donc écrire :

$$
\vartheta\left(2^{k}(\cdot)\right) f=g_{1}^{k}+g_{2}^{k}+g_{3}^{k}
$$

où

$$
\begin{gathered}
g_{1}^{k}=\vartheta\left(2^{k}(\cdot)\right) \sum_{j \geq k+3} \Delta_{j} f ; \\
g_{2}^{k}=\vartheta\left(2^{k}(\cdot)\right) \sum_{j \leq k-3} \Delta_{j} f ; \\
g_{3}^{k}=\vartheta\left(2^{k}(\cdot)\right) \Delta_{k+v} f \quad \nu=-2, \ldots, 2 . \\
\|f\|_{\left(\dot{X}_{p}^{s, q}\right)_{\ell P(z)}} \leq \sum_{i=1}^{3}\left[\sum_{k}\left\|g_{i}^{k}\right\|_{\dot{X}_{p}^{s, q}}^{p}\right]^{1 / p}=A_{1}+A_{2}+A_{3} .
\end{gathered}
$$


Estimation de $A_{1}$. Pour $j \geq k+3, \vartheta\left(2^{k}(\cdot)\right) \Delta_{j} f$ est à spectre dans $\frac{1}{4} 2^{j} \leq|\xi| \leq 42^{j}$; par le lemme 3 on obtient donc :

$$
A_{1} \leq C\left[\sum_{k}\left[\sum_{j \geq k+3} 2^{s j q}\left\|\vartheta\left(2^{k}(\cdot)\right) \Delta_{j} f\right\|_{p}^{q}\right]^{p / q}\right]^{1 / p} .
$$

Ensuite, l'hypothèse $p \geq q$ et la localisation de $L^{p}$ en norme $\ell^{p}(\mathbf{Z})$, entraînent que : $\bar{A}_{1} \leq C\|f\|_{\dot{X}_{p}^{s, q}}$.

Estimation de $A_{2}$. On observe que $g_{2}^{k}$ est à spectre dans $\frac{1}{4} 2^{k} \leq$ $|\xi| \leq 42^{k}$ donc :

$$
\begin{aligned}
\left\|g_{2}^{k}\right\|_{X_{p}^{s, q}} & \leq C 2^{s k}\left\|g_{2}^{k}\right\|_{p} \\
& \leq C 2^{(s-n / p) k}\left\|\vartheta\left(\sum_{j \leq k-3}\left(\Delta_{j} f\right)\left(2^{-k}(\cdot)\right)\right)\right\|_{p} \\
& \leq C 2^{(s-n / p) k}\|\vartheta\|_{p}\left(\sum_{j \leq k-3}\left\|\Delta_{j} f\right\|_{\infty}\right) \\
& \leq C 2^{(s-n / p) k}\left(\sum_{j \leq k-3} 2^{j(n / p-s)}\left(2^{s j}\left\|\Delta_{j} f\right\|_{p}\right)\right)
\end{aligned}
$$

(Bernstein)

L'estimation $A_{2} \leq C\|f\|_{\dot{X}_{p}^{s, q}}$, s'obtient en utilisant le fait que $s<\frac{n}{p}$ et le lemme 4 .

Estimation de $A_{3}$. La proposition 4 et la proposition 1 nous donnent :

$$
\begin{aligned}
\left\|g_{3}^{k}\right\|_{X_{p}^{s, q}} & \leq C\left\|\Delta_{k+v} f\right\|_{X_{p}^{s, q}} \\
& \leq C 2^{s k}\left\|\Delta_{k+v} f\right\|_{p}
\end{aligned}
$$

d'où :

$$
\begin{aligned}
A_{3} & \leq C\left(\sum_{k}\left(2^{s k}\left\|\Delta_{k+v}\right\|_{p}\right)^{p}\right)^{1 / p} \\
& \leq C\|f\|_{\dot{X}_{p}^{s, q}} \quad \text { car } p \geq q
\end{aligned}
$$

2. Le $\operatorname{cas} s-\frac{n}{p} \in \mathbf{R}^{+} / \mathbf{N}: m=\left[s-\frac{n}{p}\right]$

Dans ce cas on a :

$$
f=\sum_{j \in \mathbf{Z}}\left(\Delta_{j} f-\sum_{|\alpha| \leq m} \frac{1}{\alpha !}\left(\Delta_{j} f\right)^{(\alpha)}(0) \cdot x^{\alpha}\right) \quad \operatorname{dans} S^{\prime}\left(\mathbf{R}^{n}\right) .
$$


De plus, pour tout $k \in \mathbf{Z}, \sum_{j \geq k} \Delta_{j} f$ converge dans $S^{\prime}\left(\mathbf{R}^{n}\right)$; et $\sum_{j \geq k}\left(\Delta_{j} f\right)^{(\alpha)}(0)$ converge pour $|\alpha| \leq m$. On peut donc écrire :

$$
\vartheta\left(2^{k}(\cdot)\right) f=g_{4}^{k}-g_{5}^{k}+g_{6}^{k}
$$

où

$$
\begin{aligned}
g_{4}^{k} & =\sum_{j \geq k+1}\left(\vartheta\left(2^{k}(\cdot)\right) \Delta_{j} f\right) \\
g_{5}^{k} & =\sum_{j \geq k+1}\left(\Delta_{j} f\right)^{(\alpha)}(0) \cdot \vartheta\left(2^{k}(\cdot)\right) x^{\alpha}, \quad|\alpha| \leq m \\
g_{6}^{k} & =\sum_{j \leq k} \vartheta\left(2^{k}(\cdot)\right) P_{j}(f), \quad P_{j}(f)(x)=\Delta_{j} f-\sum_{|\alpha| \leq m} \frac{1}{\alpha !}\left(\Delta_{j} f\right)^{(\alpha)}(0) x^{\alpha}
\end{aligned}
$$

La majoration de $\left[\sum_{k}\left\|g_{4}^{k}\right\|_{B_{p}^{s, q}}^{p}\right]^{1 / p}$ s'obtient de la même façon que pour le cas $0<s<\frac{n}{p}$.

$g_{5}^{k}$ est à spectre dans la boule $|\xi| \leq 2^{k+1}$, donc

$$
\left\|g_{5}^{k}\right\|_{\dot{B}_{p}^{s, q}} \leq C 2^{s k}\left\|g_{5}^{k}\right\|_{p}
$$

Or

$$
\begin{aligned}
\left\|g_{5}^{k}\right\|_{p} & \leq \sum_{j \geq k+1}\left\|\left(\Delta_{j} f\right)^{(\alpha)}\right\|_{\infty}\left\|\vartheta\left(2^{k}(\cdot)\right) x^{\alpha}\right\|_{p} \\
& \leq C 2^{(-n k / p-|\alpha| k)} \sum_{j \geq k+1}\left\|\left(\Delta_{j} f\right)^{(\alpha)}\right\|_{\infty} .
\end{aligned}
$$

D'où :

$$
\left\|g_{5}^{k}\right\|_{p} \leq C 2^{(-n / p-|\alpha|) k} \sum_{j \geq k+1} 2^{j(n / p+|\alpha|)}\left\|\Delta_{j} f\right\|_{p}
$$

En utilisant, alors, le fait que $s-n / p-|\alpha|>0$, le lemme 4 et l'hypothèse $p \geq q$, on obtient :

$$
\left[\sum_{k}\left\|g_{5}^{k}\right\|_{X_{p}^{s, q}}\right]^{1 / p} \leq C\|f\|_{X_{p}^{s, q}}
$$


Finalement, $g_{6}^{k}$ est à spectre dans $|\xi| \leq 2^{k+2}$ donc :

$$
\begin{aligned}
\left\|g_{6}^{k}\right\|_{\dot{X}_{p}^{s, q}} & \leq C 2^{s k}\left\|g_{6}^{k}\right\|_{p} \\
& \leq C 2^{s k} \sum_{j \leq k}\left\|\vartheta\left(2^{k}(\cdot)\right) P_{j}(f)\right\|_{p} .
\end{aligned}
$$

Or,

$$
\left|P_{j}(f)(x)\right| \leq C \sum_{|\alpha|=m+1}|x|^{m+1}\left\|\left(\Delta_{j} f\right)^{(\alpha)}\right\|_{\infty}
$$

(Bernstein)

$$
\leq C|x|^{m+1} 2^{j(n / p+m+1)}\left\|\Delta_{j} f\right\|_{p}
$$

d'où :

$$
\left\|g_{6}^{k}\right\|_{\dot{X}_{p}^{s, q}} \leq C 2^{2 k(s-n / p-m-1)} \sum_{j \leq k} 2^{j(n / p+m+1-s)}\left(2^{s j}\left\|\Delta_{j} f\right\|_{p}\right) .
$$

L’inegalité

$$
\left[\sum_{k}\left\|g_{6}^{k}\right\|_{\dot{X}_{p}^{s, q}}^{p}\right]^{1 / p} \leq C\|f\|_{\dot{X}_{p}^{s, q}}
$$

s'obtient en combinant le fait que $s-\frac{n}{p}-m-1<0$, le lemme 4 et l'hypothèse $p \geq q$.

B. Le cas $p \leq q$

Le cas $-\frac{n}{p^{\prime}}<s<\frac{n}{p}$ se déduit, par dualité, de la partie A. En effet, si $p^{\prime}=+\infty$ alors $p=1$ et évidemment $\left(\dot{X}_{1}^{s, q}\right)_{\ell^{1}(Z)} \subseteq \dot{X}_{1}^{s, q}$. Si $p^{\prime}<+\infty$ alors $q^{\prime}<+\infty$; donc le dual de $\dot{X}_{p^{\prime}}^{-s, q^{\prime}}$ est $\dot{X}_{p}^{s, q}$. De plus, de A on tire :

$$
\dot{X}_{p^{\prime}}^{-s, q^{\prime}} \subseteq\left(\dot{X}_{p^{\prime}}^{-s, q^{\prime}}\right)_{\ell^{p^{\prime}}(\mathbf{Z})}
$$

de sorte que l'inclusion $\left(\dot{X}_{p}^{s, q}\right)_{\ell p(Z)} \subseteq \dot{X}_{p}^{8, q}$ se déduit en remarquant que le dual de $\ell^{p^{\prime}}\left(\mathbf{Z}, \dot{X}_{p^{\prime}}^{-s, q^{\prime}}\right)$ est $\ell^{p}\left(\mathbf{Z}, \dot{X}_{p}^{s, q}\right)$.

Il nous reste à montrer que $\left(\dot{X}_{p}^{s, q}\right)_{\ell p(Z)} \subseteq \dot{X}_{p}^{s, q}$ pour $-s-\frac{n}{p} \in \mathbf{R}^{+} / \mathbf{N}$. Pour ce faire, soit $f \in\left(\dot{X}_{p}^{s, q}\right)_{\ell p(Z)}$ alors, l'identification de $\dot{X}_{p}^{s, q}$ à un sous-espace de $D^{\prime}\left(\mathbf{R}_{0}^{n}\right)$, nous ramène à montrer que :

$$
g=\sum_{j \in \mathbf{Z}}\left(\eta\left(2^{j}(\cdot)\right) f\right) \in \dot{X}_{p}^{s, q} .
$$




\section{Estimation de la norme}

Choisissons $\lambda$ vérifiant les hypothèses du lemme 5 , portée par $|x| \leq 1$ et $\lambda \in D\left(\mathbf{R}^{n}\right)$.

$$
\begin{aligned}
L_{j}(g) & =\sum_{k \leq j-2} L_{j}\left(\eta\left(2^{k}(\cdot)\right) f\right)+L_{j}\left(\sum_{k \geq j-1} \eta\left(2^{k}(\cdot)\right) f\right) \\
& =h_{j}^{1}+h_{j}^{2}, \\
\|g\|_{\dot{B}_{p}^{s, q}} & \leq C\left[\left(\sum_{j} 2^{s j q}\left\|h_{j}^{1}\right\|_{p}^{q}\right)^{1 / q}+\left(\sum_{j} 2^{s j q}\left\|h_{j}^{2}\right\|_{q}^{q}\right)^{1 / q}\right] \\
& \leq E+F .
\end{aligned}
$$

Pour $k \leq j-2, L_{j}\left(\eta\left(2^{k}(\cdot)\right) f\right)$ est portée par $2^{-k-2} \leq|x| \leq$ $2^{-k+2}$; donc, le lemme 1 et la localisation de $L^{p}$ en norme $\ell^{p}(\mathbf{Z})$ nous donnent :

$$
\left\|h_{j}^{1}\right\|_{p} \leq C\left[\sum_{k \leq j-2}\left\|L_{j}\left(\eta\left(2^{k}(\cdot)\right) f\right)\right\|_{p}^{p}\right]^{1 / p} .
$$

De là, l'hypothèse $p \leq q$ entraîne, aussitôt, que $E$ est majorée par

$$
C\left[\sum_{k}\left\|\eta\left(2^{k}(\cdot)\right) f\right\|_{\dot{X}_{p}^{s, q}}^{p}\right]^{1 / p} .
$$

$\left\|h_{j}^{2}\right\|_{p}$ est majorée par $\sum_{k \geq j-1}\|\lambda\|_{1}\left\|\eta\left(2^{k}(\cdot)\right) f\right\|_{p}$, donc

$$
F \leq C\left[\sum_{j} 2^{s j q}\left(\sum_{k \geq j-1}\left\|\eta\left(2^{k}(\cdot)\right) f\right\|_{p}\right)^{q}\right]^{1 / q} .
$$

En combinant le lemme 4, l'hypothèse $s>0$ et le lemme 6 , on obtient :

$$
F \leq C\|f\|_{\left(\dot{X}_{p}^{s, q}\right)_{\ell p(z)}} \text {. }
$$

\section{Appartenance à la réalisation : $m=\left[s-\frac{n}{p}\right]$}

On pose : $f_{j}=\eta\left(2^{j}(\cdot)\right) f$, alors

- $f_{j}$ de classe $C^{m}$;

- $f_{j}^{(\alpha)}(0)=0$ pour $|\alpha| \leq m$;

- $\left|f_{j}^{(\alpha)}(x)\right| \leq C\left\|f_{j}\right\|_{X_{p}^{s, q}}|x|^{(s-n / p-m)}$ pour $|\alpha|=m$. 
On a alors, $\left|f_{j}^{(\alpha)}(x)\right| \leq C\|f\|_{\left(\dot{X}_{p}^{s, q}\right)_{\ell p(\mathbf{z})}}|x|^{(s-n / p-|\alpha|)}$ pour $|\alpha| \leq$ $m$. De plus, pour tout $x \in \mathbf{R}^{n}$, le nombre de $j \in \mathbf{Z}$, tel que $x \in \operatorname{supp} f_{j}$, est inférieur à 4 ; d'où :

$$
\sum_{j \in \mathbf{Z}}\left|f_{j}^{(\alpha)}(x)\right| \leq C|x|^{(s-n / p-|\alpha|)} \quad \text { pour }|\alpha| \leq m .
$$

En particulier, $\sum_{j \in \mathbf{Z}} f_{j}^{(\alpha)}$ converge uniformément sur tout compact de $\mathbf{R}^{n}$ pour $|\alpha| \leq m$; donc $g=\sum_{j \in \mathbf{Z}} f_{j}$ est de classe $C^{m} ; g^{(\alpha)}(0)=0$ pour $|\alpha| \leq m$ et $\left|g^{(\alpha)}\right| \leq C|x|^{(s-n / p-m)}$ pour $|\alpha|=m$.

IV. Contre-exemples. Nous allons montrer que $\dot{X}_{p}^{s, q}$ n'est localisable en aucune norme $\ell^{t}(\mathbf{Z})$ pour $p \neq q$. D'après le corollaire 2 , il nous suffit de trouver un réel $t$ tel q'on n'ait ni $\dot{X}_{p}^{s, q} \subseteq\left(\dot{X}_{p}^{s, q}\right)_{\ell^{t}(z)}$, ni $\left(\dot{X}_{p}^{s, q}\right)_{\ell^{t}(\mathbf{z})} \subseteq \dot{X}_{p}^{s, q}$.

Pour cela, considérons une fonction $\psi \in S\left(\mathbf{R}^{n}\right)$ positive, à spectre dans $|\xi| \leq \frac{1}{4}, \psi \neq 0$ sur $\frac{1}{2} \leq|x| \leq 2$ et telle que $\psi^{(\alpha)}(0)=0$ pour $|\alpha| \leq N(N$ assez grand), (la construction de cette fonction est la même que celle du lemme 7 ).

On pose :

$$
\begin{aligned}
& f=\sum_{j \leq 0} \alpha_{j} \psi\left(2^{j} x\right) 2^{n j / p} e^{i x_{1}} \\
& g=\sum_{j \geq 0} \beta_{j} 2^{-s j} \psi\left(2^{j} x\right) 2^{n j / p} e^{i 2^{j} x_{1}}
\end{aligned}
$$

$\left(\alpha_{j}\right)_{\ell \in Z}$ et $\left(\beta_{j}\right)_{j \in Z}$ sont deux suites de réels positifs, choisies de telle façon que les deux séries convergent dans $S^{\prime}\left(\mathbf{R}^{n}\right)$ (par exemple : $\left(\alpha_{j}\right)_{j} \in \ell^{\infty}(\mathbf{Z})$ et $\left(\beta_{j}\right)_{j} \in$ $\ell^{\infty}(\mathbf{Z})$. On a $\Delta_{0} f=f$ est à spectre dans $\frac{3}{4} \leq|\xi| \leq \frac{5}{4}$ et

$$
\Delta_{j} g=\beta_{j} 2^{-s j} \psi\left(2^{j} x\right) 2^{n j / p} e^{i 2^{j} x_{1}} .
$$

De plus, $f^{(\gamma)}(0)=0$ et $\left(\Delta_{j}(g)\right)^{(\gamma)}(0)=0$ pour $|\gamma| \leq N$. D'où on tire : $f \in \dot{X}_{p}^{s, q}$ (resp. $g \in \dot{X}_{p}^{s, q}$ ) si et seulement si $f \in \dot{B}_{p}^{s, q}$ (resp. $g \in \dot{B}_{p}^{s, q}$ ).

Nous allons prouver :

$$
\begin{aligned}
& \|f\|_{B_{p}^{s, q}} \approx\left[\sum_{j \leq 0} \alpha_{j}^{p}\right]^{1 / p} \\
& \|f\|_{\left(\dot{X}_{p}^{s, q}\right)_{\ell^{t}(z)}} \approx\left[\sum_{j \leq 0} \alpha_{j}^{t}\right]^{1 / t}, \\
& \|f\|_{B_{p}^{s, q}} \approx\left[\sum_{j \geq 0} \beta_{j}^{q}\right]^{1 / q}, \\
& \|g\|_{\left(\dot{X}_{p}^{s, q}\right)_{\ell^{t}(z)}} \approx\left[\sum_{j \geq 0} \beta_{j}^{t}\right]^{1 / t} .
\end{aligned}
$$

Ces propriétés étant admises, nous choisissons $\left(\alpha_{j}\right)_{j},\left(\beta_{j}\right)_{j}$ et $t$ : 
a. Le cas $p<q$

On prend $t \in] p, q\left[, \alpha_{j}=|j|^{-1 / p}\right.$ et $\beta_{j}=|j|^{-1 / t}$; alors $f \in\left(\dot{X}_{p}^{s, q}\right)_{\ell^{t}(Z)}$ et $f \notin \dot{X}_{p}^{s, q} ; g \notin\left(\dot{X}_{p}^{s, q}\right)_{\ell^{t}(z)}$ et $g \in \dot{X}_{p}^{s, q}$.

\section{b. Le cas $p>q$}

On prend $t \in] q, p\left[, \alpha_{j}=|j|^{-1 / t}\right.$ et $\beta_{j}=|j|^{-1 / q} ;$ on obtient $f \notin\left(\dot{X}_{p}^{s, q}\right)_{\ell^{t}(z)}$ et $f \in \dot{X}_{p}^{s, q} ; g \in\left(\dot{X}_{p}^{s, q}\right)_{\ell^{t}(\mathbf{Z})}$ et $g \notin \dot{X}_{p}^{s, q}$.

Pour montrer les quatre propriétés, on a besoin du lemme suivant :

Lemme 8. Pour tout $p \in[1,+\infty]$ et toutes fonctions $h$ et $\vartheta$ de $S\left(\mathbf{R}^{n}\right)$ telles que $h^{(\gamma)}(0)=\vartheta^{(\gamma)}(0)=0$ pour $|\gamma| \leq N$, la suite $\left(2^{|v| N}\left\|h\left(2^{v}(\cdot)\right) \vartheta\right\|_{p}\right)_{v \in \mathbf{Z}}$ appartient à $\ell^{1}(\mathbf{Z})$.

Preuve : Il suffit de remarquer que $|\vartheta(x)| \leq C|x|^{N+1}$ et $|h(x)| \leq$ $C|x|^{N+1}$.

$\triangle$ Preuve du $\left(1^{\circ}\right)$. On a $\|f\|_{\dot{B}_{p}^{s, q}}=\left\|\Delta_{0} f\right\|_{p}=\|f\|_{p} ;$ de plus la proposition 2 et l'hypothèse sur $\psi$ nous donnent :

$$
\|f\|_{p} \approx\left[\sum_{v \in \mathbf{Z}}\left\|\psi\left(2^{v}(\cdot)\right) f\right\|_{p}^{p}\right]^{1 / p}
$$

Or $\left\|\psi\left(2^{v}(\cdot)\right) f\right\|_{p} \leq \sum_{j \leq 0} \alpha_{j}\left\|\psi\left(2^{v-j}(\cdot)\right) \psi\right\|_{p}$; on utilise le lemme 8 et l'inegalité de Young; on obtient :

$$
\|f\|_{\dot{B}_{p}^{s, q}}=\|f\|_{p} \leq C\left[\sum_{j \leq 0} \alpha_{j}^{p}\right]^{1 / p}
$$

Inversement, de la positivité de $\psi$ et $\left(\alpha_{j}\right)$, on tire :

$$
\left|\psi\left(2^{v} x\right) f(x)\right| \geq \alpha_{v}\left(\psi\left(2^{v} x\right)\right)^{2} 2^{n v / p}
$$

d'où

$$
\begin{aligned}
\|f\|_{p} & \geq\left[\sum_{v \leq 0}\left\|\psi\left(2^{v}(\cdot)\right) f\right\|_{p}^{p}\right]^{1 / p} \\
& \geq C\left[\sum_{j \leq 0} \alpha_{j}^{p}\right]^{1 / p}
\end{aligned}
$$


$\triangleright$ Preuve du $\left(2^{\circ}\right)$

$$
\|f\|_{\left(\dot{X}_{p}^{s, q}\right)_{\ell^{t}(\mathbf{Z})}} \approx\left[\sum_{v \in Z}\left\|\psi\left(2^{v}(\cdot)\right) f\right\|_{\dot{X}_{p}^{s, q}}^{t}\right]^{1 / t} .
$$

Pour $v \leq-1, \psi\left(2^{v}(\cdot)\right) f$ est à spectre dans $\frac{7}{8} \leq|\xi| \leq \frac{11}{8}$, donc

$$
\left\|\psi\left(2^{v}(\cdot)\right) f\right\|_{\dot{B}_{p}^{s, q}} \geq C\left\|\psi\left(2^{v}(\cdot)\right) f\right\|_{p} \quad \text { (lemme 3); }
$$

d'où :

$$
\begin{aligned}
\|f\|_{\left(\dot{X}_{p}^{s, q}\right)_{\ell^{t}(\mathbf{z})}} & \geq C\left[\sum_{v \leq 0}\left\|\psi\left(2^{v-1}(\cdot)\right) f\right\|_{p}^{t}\right]^{1 / t} \\
& \geq C\left[\sum_{j \leq 0} \alpha_{j}^{t}\right]^{1 / t}
\end{aligned}
$$

Inversement, si $v \leq 0,\left\|\psi\left(2^{v}(\cdot)\right) f\right\|_{\dot{B}_{p}^{s, q}} \leq C\left\|\psi\left(2^{v}(\cdot)\right) f\right\|_{p}$, donc

$$
\left\|\psi\left(2^{v}(\cdot)\right) f\right\|_{\dot{X}_{p}^{s, q}} \leq C \sum_{j \leq 0} \alpha_{j}\left\|\psi\left(2^{v-j}(\cdot)\right) \psi\right\|_{p} .
$$

Si $v \geq 0$,

$$
\begin{aligned}
\left\|\psi\left(2^{v}(\cdot)\right) f\right\|_{\dot{X}_{p}^{s, q}} & \leq C 2^{s v}\left\|\psi\left(2^{v}(\cdot)\right) f\right\|_{p} \\
& \leq C \sum_{j \leq 0} \alpha_{j} 2^{s v}\left\|\psi\left(2^{v-j}(\cdot)\right) \psi\right\|_{p}
\end{aligned}
$$

On obtient alors, pour tout $v \in \mathbf{Z}$ :

$$
\left\|\psi\left(2^{v}(\cdot)\right) f\right\|_{\dot{X}_{p}^{s, q}} \leq C \sum_{j \leq 0} \alpha_{j} 2^{s|v-j|}\left\|\psi\left(2^{v-j}(\cdot)\right) \psi\right\|_{p}
$$

de sorte que le lemme 8 et l'inegalité de Young nous donnent :

$$
\|f\|_{\left(\dot{X}_{p}^{s, q}\right)_{\ell^{t}(\mathbf{z})}} \leq C\left[\sum_{j \leq 0} \alpha_{j}^{t}\right]^{1 / t}
$$

D Preuve du $\left(3^{\circ}\right)$. C'est une conséquence du lemme 3, car les fonctions $\psi\left(2^{j} x\right) e^{i 2^{j} x_{1}}$ sont à spectre dans $\frac{3}{4} 2^{j} \leq|\xi| \leq \frac{5}{4} 2^{j}$.

$\triangleright$ Preuve du $\left(4^{\circ}\right)$.

$$
\|g\|_{\left(\dot{X}_{p}^{s, q}\right)_{\ell^{t}(z)}} \approx\left[\sum_{v \in Z}\left\|\psi\left(2^{v}(\cdot)\right) f\right\|_{\dot{X}_{p}^{s, q}}^{t}\right]^{1 / t} .
$$


D'après le lemme $3,\left\|\psi\left(2^{v}(\cdot)\right) f\right\|_{\dot{X}_{p}^{s, q}}$ est majorée par :

$$
\begin{aligned}
& C\left[\left(\sum_{j \geq v} \beta_{j}^{q}\left\|\psi\left(2^{v-j}(\cdot)\right) \psi\right\|_{p}^{q}\right)^{1 / q}+2^{s v}\left\|\sum_{j \leq v} 2^{-s j} \beta_{j} \psi\left(2^{v}(\cdot)\right) \psi\left(2^{j}(\cdot)\right) 2^{n j / p}\right\|_{p}\right] \\
= & G+H .
\end{aligned}
$$

Or,

$$
G \leq C \sum_{j \geq 0} \beta_{j}\left\|\psi\left(2^{v-j}(\cdot)\right) \psi\right\|_{p}
$$

et

$$
H \leq C \sum_{j \geq 0} \beta_{j} 2^{|v-j| s}\left\|\psi\left(2^{v-j}(\cdot)\right) \psi\right\|_{p}
$$

de sorte que par le lemme 8 et l'inegalité de Young, on obtient :

$$
\|g\|_{\left(\dot{X}_{p}^{s, q}\right)_{\ell^{t}(\mathbf{z})}} \leq C\left[\sum_{j \geq 0} \beta_{j}^{t}\right]^{1 / t} .
$$

Inversement, pour $v \geq 0$, on a :

$$
\Delta_{v+3}\left(\psi\left(2^{v}(\cdot)\right) g\right)=2^{n(v+3) / p} \beta_{v+3} 2^{-s(v+3)} \psi\left(2^{v}(\cdot)\right) \psi\left(2^{v+3}(\cdot)\right) e^{i 2^{v+3} x_{1}}
$$

donc $\left\|\psi\left(2^{v}(\cdot)\right) g\right\|_{\dot{X}_{p}^{s, q}} \geq C \beta_{v+3}\left\|\psi \cdot \psi\left(2^{3}(\cdot)\right)\right\|_{p} ;$ ce qui montre que $\|g\|_{\left(\dot{X}_{p}^{s, q}\right)_{\ell^{\ell}(z)}}$ est minorée par $C\left[\sum_{j \geq 0} \beta_{j}^{t}\right]^{1 / t}$.

V. Problème des multiplicateurs. On note par $\mathcal{E}\left(\mathbf{R}_{0}^{n}\right)$, l'espace des fonctions de classe $C^{\infty}$ sur $\mathbf{R}_{0}^{n}$. Toute distribution $u \in D^{\prime}\left(\mathbf{R}_{0}^{n}\right)$ définit une application linéaire $M_{u}$ de $\mathcal{E}\left(\mathbf{R}_{0}^{n}\right)$ dans $D^{\prime}\left(\mathbf{R}_{0}^{n}\right)$ par : pour $f \in \mathcal{E}\left(\mathbf{R}_{0}^{n}\right)$ et $g \in D\left(\mathbf{R}_{0}^{n}\right)$ :

$$
\left\langle M_{u}(f), g\right\rangle=\langle f u, g\rangle=\langle u, f g\rangle \text {. }
$$

Considérons un E.B.D. $\left(\mathbf{R}_{0}^{n}\right), E$ contenant $D\left(\mathbf{R}_{0}^{n}\right)$ comme sous-espace; on note par $E_{\infty}$ l'adhérence de $\mathcal{E}\left(\mathbf{R}_{0}^{n}\right) \cap E$ dans $E$.

On dit qu'une distribution $u \in D^{\prime}\left(\mathbf{R}_{0}^{n}\right)$ est un multiplicateur de $E$, s'il existe $C>0$ tel que pour tout $f \in \mathcal{E}\left(\mathbf{R}_{0}^{n}\right) \cap E$, on ait : $f u \in E$ et $\|f u\|_{E} \leq C\|f\|_{E}$. Alors $M_{u}$ se prolonge en un opérateur linéaire, continu de $E_{\infty}$ dans $E$. Notons par $M(E)$ l'espace des multiplicateurs de $E$; muni de la norme $\left\|M_{u}\right\|_{\mathcal{L}\left(E_{\infty}, E\right)}$, $M(E)$ est un E.B.D. $\left(\mathbf{R}_{0}^{n}\right)$.

Supposons que l'espace $E$ est un $D\left(\mathbf{R}_{0}^{n}\right)$-module homogène; on peut vérifier facilement que $M(E)$ est un $D\left(\mathbf{R}_{0}^{n}\right)$-module et homogène de degré 0 . De plus :

Proposition 5. Si $E$ est localisable en norme $\ell^{p}(\mathbf{Z}), 1 \leq p \leq+\infty$, alors $M(E)$ est localisable en norme $\ell^{\infty}(\mathrm{Z})$. 
La preuve est analogue à celle de [2].

Désignons par $\left(B_{p}^{s, q}\right)_{\ell^{\infty}(\mathbf{z})}$, l'espace des distributions $m \in D^{\prime}\left(\mathbf{R}_{0}^{n}\right)$ telles que : $\sup _{j \in \mathbf{Z}}\left\|\eta \cdot d_{2^{j}} m\right\|_{B_{p}^{*, q}}<+\infty$. Le théorème 1 s'étend aux espaces $\dot{X}_{p}^{s, p}$ de la façon suivante :

Théorème 3. Si $s-\frac{n}{p} \in \mathbf{R}^{+} / \mathbf{N}$ alors $M\left(\dot{X}_{p}^{s, p}\right)=\left(B_{p}^{s, p}\right)_{\ell^{\infty}(\mathbf{Z})}$.

Preuve : Le théorème est démontré par E. Sawyer dans le cas particulier où $p=2, s-\frac{n}{2} \in \mathbf{R}^{+} / \mathbf{N}$ et $\frac{s-n}{2} \in \mathbf{R}^{+} / \mathbf{N}$.

Soit $m \in M\left(\dot{X}_{p}^{s, p}\right)$, d'après le lemme 7 on a $: \eta \cdot d_{2^{j}} m \in L^{p}$ et $\left\|\eta \cdot d_{2^{j}} m\right\|_{p} \leq$ $C\left\|\eta \cdot d_{2^{j}} m\right\|_{\dot{B}_{p}^{s, p}}$. De plus, $\left\|\eta \cdot d_{2^{j}} m\right\|_{\dot{B}_{p}^{s, p}} \leq\|m\|_{M\left(\dot{X}_{p}^{s, p}\right)}\|\eta\|_{\dot{X}_{p}^{s, p} ; \text { or, }}$

$$
\left\|\eta \cdot d_{2^{j}} m\right\|_{B_{p}^{*, p}} \leq C\left[\left\|\eta \cdot d_{2^{j}} m\right\|_{B_{p}^{s, p}}+\left\|\eta \cdot d_{2^{j}} m\right\|_{p}\right]
$$

donc $\sup _{j \in Z}\left\|\eta \cdot d_{2^{j}} m\right\|_{B_{p}^{*, p}}<+\infty$.

Réciproquement, soit $m \in\left(B_{p}^{s, p}\right)_{\ell \infty(Z)}$, pour montrer que $m \in M\left(\dot{X}_{p}^{s, p}\right)$, il nous suffit, d'après le théorème 2 et la proposition 7 , de montrer que $m \in$ $\left(M\left(\dot{X}_{p}^{s, p}\right)\right)_{\ell \infty(Z)}$.

Soit $\chi \in D\left(\mathbf{R}_{0}^{n}\right)$ telle que $\chi=1$ sur le support de $\eta$; pour $f \in \dot{X}_{p}^{s, p}$ on a :

$$
\left\|\eta \cdot d_{2^{j}} m \cdot f\right\|_{B_{p}^{s, p}} \leq C\left\|\eta \cdot d_{2^{j}} m \cdot f\right\|_{B_{p}^{*, p}}
$$

or, $B_{p}^{s, p}$ est une algèbre $\left(\operatorname{car} s>\frac{n}{p}\right)$, donc

$$
\left\|\eta \cdot d_{2^{j}} m \cdot f\right\|_{\dot{B}_{p}^{s, p}} \leq C\left\|\eta \cdot d_{2^{j}} m\right\|_{B_{p}^{*, p}}\|\chi \cdot f\|_{B_{p}^{*, p}}
$$

Ce qui prouve que $\eta \cdot d_{2^{j}} m \cdot f \in \dot{B}_{p}^{s, p}$ et, est portée par $\frac{1}{2} \leq|x| \leq 2$, donc $\eta \cdot d_{2} ; m \cdot f \in \dot{X}_{p}^{s, p}$.

VI. Inégalité de Hardy. Soit $E$ un E.B.D. $\left(\mathbf{R}_{0}^{n}\right)$, supposé homogène de degré $\alpha$ et qui est $D\left(\mathbf{R}_{0}^{n}\right)$-module.

Proposition 6. S'il existe $C>0$, tel que pour toute $f \in E$ portée par $\frac{1}{2} \leq|x| \leq 2$ on a $\|f\|_{p} \leq C\|f\|_{E}$, alors pour toute $g \in(E)_{\ell p(Z)}$ on a $g \in L^{p}\left(|x|^{-n+\alpha p} d x\right)$ et :

$$
\left[\int_{\mathbf{R}^{n}}|g(x)|^{p}|x|^{-n+\alpha p} d x\right]^{1 / p} \leq C\|g\|_{(E)_{\ell>(z)}} .
$$


Preuve : De la localisation de $L^{p}$ en norme $\ell^{p}(\mathbf{Z})$ on tire :

$$
\left[\int_{\mathbf{R}^{n}}|g(x)|^{p}|x|^{-n+\alpha p} d x\right]^{1 / p} \leq C\left[\sum_{j \in \mathbf{Z}} \int\left|\eta\left(2^{j} x\right) g(x)\right|^{p}|x|^{-n+\alpha p} d x\right]^{1 / p}
$$

Or, $|x| \approx 2^{-j}$ dans le support de $\eta\left(2^{j}(\cdot)\right)$, donc :

$$
\begin{aligned}
{\left[\int_{\mathbf{R}^{n}}|g(x)|^{p}\right.} & \left.|x|^{-n+\alpha p} d x\right]^{1 / p} \\
& \leq C\left[\sum_{j \in \mathbf{Z}} 2^{j(n-\alpha p)}\left\|\eta\left(2^{j}(\cdot)\right) g\right\|_{p}^{p}\right]^{1 / p} \\
& \leq C\left[\sum_{j \in \mathbf{Z}} 2^{-j \alpha p}\left\|\eta \cdot g\left(2^{-j}(\cdot)\right)\right\|_{p}^{p}\right]^{1 / p} \\
& \leq C\left[\sum_{j \in \mathbf{Z}} 2^{-j \alpha p}\left\|\eta \cdot g\left(2^{-j}(\cdot)\right)\right\|_{E}^{p}\right]^{1 / p} \\
& \leq C\|g\|_{(E)_{\ell p}(\mathbf{Z})} .
\end{aligned}
$$

De la proposition 6 , du théorème 2 et du lemme 6 résulte une inégalité du type de celle de Hardy-Littlewood [8] :

Proposition 7. Pour $0<s<\frac{n}{p}$ ou $s-\frac{n}{p} \in \mathbf{R}^{+} / \mathbf{N}$ et $p \geq q$, on a l'inégalité de Hardy : pour toute $f \in \dot{X}_{p}^{s, q}$ :

$$
\left[\int_{\mathbf{R}^{n}}|f(x)|^{p}|x|^{-s p} d x\right]^{1 / p} \leq C\|f\|_{\dot{X}_{p}^{s, q}} .
$$

Par contre, nous allons montrer que cette inégalité n'est pas forcément vérifiée quand $p<q$. Pour ce faire, considérons une fonction $\psi \in S\left(\mathbf{R}^{n}\right)$ positive, à spectre dans $|\xi| \leq 1, \psi^{(\gamma)}(0)=0$ pour $|\gamma| \leq N(N$ un entier assez grand) et $\psi \neq 0$ sur le support de $n$. On pose $f=\sum_{j \geq 1} j^{-1 / p} 2^{-s j} 2^{n j / p} \psi\left(2^{j} x\right)$; cette série est convergente dans $S^{\prime}\left(\mathbf{R}^{n}\right)$. De plus :

$\left(1^{\circ}\right)$ En combinant la proposition 2, le lemme 8 et l'inégalité de Young on obtient :

$$
\begin{aligned}
\|f\|_{p} & \approx\left[\sum_{k \in \mathbf{Z}}\left\|\psi\left(2^{k}(\cdot)\right) f\right\|_{p}^{p}\right]^{1 / p} \\
& \leq C\left[\sum_{j \geq 1} \frac{2^{-s j p}}{j}\right]^{1 / p}<+\infty
\end{aligned}
$$


$\left(2^{\circ}\right)$ Si $s-\frac{n}{p} \in \mathbf{R}^{+} / \mathbf{N}$ et $|\gamma| \leq m\left(m=\left[s-\frac{n}{p}\right]\right)$ alors la série

$$
\sum_{j \geq 1} j^{-1 / p} 2^{-s j} 2^{n j / p} 2^{j|\gamma|} \psi^{(\gamma)}\left(2^{j} x\right)
$$

converge uniformément sur $\mathbf{R}^{n}$; en particulier $f$ est de classe $C^{m}$ et $f^{(\gamma)}(0)=0$ pour $|\gamma| \leq m$.

$\left(3^{\circ}\right)$ Par le lemme 3 on a $\|f\|_{B_{p}^{s, q}} \leq C\left[\sum_{j \geq 1} j^{-q / p}\right]^{1 / q}<+\infty$.

Ces propriétés montrent que $f \in \dot{X}_{p}^{s, q}$; par contre on a :

$$
|f(x)|^{p}=(f(x))^{p} \geq \sum_{j \geq 1} \frac{1}{j} 2^{-s j p} 2^{n j}\left(\psi\left(2^{j} x\right)\right)^{p},
$$

d'où :

$$
\begin{aligned}
\int_{\mathbf{R}^{n}}|f(x)|^{p}|x|^{-s p} d x & \geq \sum_{j \geq 1} \frac{1}{j} \int_{\mathbf{R}^{n}}\left|2^{j} x\right|^{-s p}\left|\psi\left(2^{j} x\right)\right|^{p} 2^{n j} d x \\
& \geq C \sum_{j \geq 1} \frac{1}{j}=+\infty
\end{aligned}
$$

\section{REFERENCES}

[1] G. Bourdaud, Analyse fonctionnelle dans l'espace Euclidien, Publ. Math. Univ. Paris VII (1987).

[2] G. Bourdaud, Localisations des espaces de Besov, Studia Math. (a paraitre).

[3] G. Bourdaud, Réalisations des espaces de Besov homogènes (I), Prépubl. 86T25, Univ. de Paris-Sud, Dép. Math., Bât. 425, 91405 Orsay.

[4] G. Bourdaud, Réalisations des espaces de Besov homogènes (II), Arkiv für Mat. (a paraître).

[5] B. MUCKENHOUPT, R. L. WHEEdEN \& W. S. YOUNG, $L^{2}-m u l t i p l i e r s$ with power weights, Adv. in Math. 49 (1983), 170-216.

[6] J. Peetre, New thoughts on Besov spaces, Duke Univ. Math. Series I Duke Univ., Durham, North Carolina (1976).

[7] E. SAWYER, Multipliers of Besov and power-weighted $L^{2}$ spaces, Indiana Univ. Math. J. 33 (1984), 353-366.

[8] E. M. STEIN \& G. WEISS, Fractional integrals on n-dimensional Euclidean space, J. Math. Mech. 7 (1958), 503-514.

[9] R. S. Strichartz, Multipliers on fractional Sobolev spaces, J. Math. Mech. 19 (1967), 1031-1060.

[10] H. Triebel, Theory of Functional Spaces, Akad. Verlag. Geest und Portig K., Leipzig (1983).

U.F.R. de Mathématiques

Université Paris VII

Tour 45-55, 5ème étage

2, place Jussieu

75251 Paris Cédex 05

Received June 22, 1987.
Département de Mathématiques

Université d'Orléans

45067 Orléans Dédex

France 